\title{
Ethnologies
}

\section{Jouer et raconter en ligne}

Une affirmation des identités sous la contrainte

\section{Karim Chibout et Martial Martin}

Volume 32, numéro 1, 2010

Jouer

Play

URI : https://id.erudit.org/iderudit/045213ar

DOI : https://doi.org/10.7202/045213ar

Aller au sommaire du numéro

Éditeur(s)

Association Canadienne d'Ethnologie et de Folklore

ISSN

1481-5974 (imprimé)

1708-0401 (numérique)

Découvrir la revue

Citer cet article

Chibout, K. \& Martin, M. (2010). Jouer et raconter en ligne : une affirmation des identités sous la contrainte. Ethnologies, 32(1), 71-86.

https://doi.org/10.7202/045213ar
Résumé de l'article

Raconter des histoires autour de son usage des jeux massivement multijoueurs s'apparente en premier lieu à une imprégnation socioculturelle, à un hommage du joueur à cet univers ludique persistant. Cependant, la fanfic peut aussi s'opposer au jeu et rompre avec la continuité de l'univers pour affirmer une identité propre au joueur et des relations sociales singulières dans le cadre de son équipe (guilde). Les éditeurs des jeux tolèrent ce braconnage narratif et la diversité des « trajectoires d'usage " parce qu'ils ne remettent pas fondamentalement en cause l'unité du jeu. Les fanfictions profiteraient même au jeu qui, inexorablement, fait ventre de tout en utilisant les récits comme des extensions officieuses propices à son expansion. 


\section{JOUER ET RACONTER EN LIGNE}

Une affirmation des identités sous la contrainte

Karim Chibout

Martial Martin

IUT de Troyes, Université de Reims

Ce qui a créé l'humanité, c'est la narration.

Pierre Janet

\section{Introduction}

Au sein de l'univers des jeux, les jeux vidéo apparaîtront sans doute comme ceux qui sont les moins aptes à laisser s'exprimer la liberté personnelle, qui semble prisonnière du code ou du programme. On aurait peine à reconnaître dans ce champ les terrains de luttes et de contestations qu'ont pu historiquement représenter les sports. Jouer un jeu vidéo, c'est d'abord consommer passivement un produit culturel, subir la vision du monde des grands studios de production. Cependant, l'observation d'activités qu'on désignera d'abord comme périludiques (avant de s'apercevoir de leur caractère central dans l'usage d'un jeu), telles que l'écriture de "fanfictions ", c'est-à-dire l'invention et le partage d'histoires autour des univers et des personnages des jeux, laisse entrevoir une capacité insoupçonnée d'interprétation et d'opposition aux règles établies dans une vision du monde différente qui met en scène une construction de l'individuel dans ses rapports au collectif. Il s'agira, pour nous, d'étudier spécifiquement les modalités de l'affirmation du joueur dans l'activité du récit qu'il soit personnel ou commun à un groupe (une équipe ou une "guilde») dans le cadre de l'usage des MMORPG ou jeux de rôle en ligne massivement multijoueur, à partir de l'observation d'une cinquantaine de sites et de la rencontre de joueurs/ écrivains de fanfictions dans le cadre de «focusgroups » constitués d'étudiants. Nous essaierons, donc, de cerner comment la pratique duale du jeu et de l'écriture permet l'expression dynamique d'un tribalisme moderne complexe (à l'œuvre dans et hors le jeu) ; puis nous essaierons 
de comprendre comment une activité que l'on jugerait de prime abord comme «antijeu» permet de maintenir l'univers du jeu.

\section{Les récits de joueurs, des recréations tribales}

Tout environnement social est une perpétuelle fabrique de sens; l'univers virtuel n'échappe pas à cette règle. L'individu qui intègre le jeu le fait par "génération spontanée »; il crée de quelques pièces son personnage, assimile des règles ( $\mathrm{du} \mathrm{jeu}$, de conduite, stratégiques) et installe des liens sociaux avec d'autres joueurs. De la foule d'anonymes virtuels aux petites communautés de joueurs (guildes), il existe une variété dans le degré et la durée des attachements. Les groupements de joueurs s'apparentent aux «tribus » modernes caractérisées "par les rassemblements ponctuels et l'éparpillement » (Maffesoli 1988: 137) et donc par leur caractère précaire; précarité virtuelle sans doute accentuée par le peu de canaux de communication à l'œuvre (en regard de la communication en vis-à-vis) et de ce fait par la pauvreté du contexte social propre au jeu. Pour sortir des agrégats hasardeux et instables au fil des parties, les joueurs se fabriquent une histoire pour gagner en signification et faire de leur groupe une "communauté d'adhérence » (Chibout et Martin 2009). Les guildes disposent de fandoms, lieux supplémentaires de coprésence groupale (auxquels s'ajoutent parfois des rencontres IRL), dans lesquels les gamers publient des fanfics, des fanarts et chantent des louanges à la gloire de leur petite communauté.

Parmi les fictions proposées, certaines sont au plus près du jeu, des reportages au cœur des raids et des combats ou des récits détaillés de quêtes réussies. Le lecteur s'approprie naturellement l'histoire parce qu'elle reflète une quête passée ou évoque des souvenirs d'actions de jeu ; il fait sienne cette histoire parce qu'elle renvoie à du probablement vécu ou à du possiblement à vivre dans le jeu. Le jeu et le récit qui lui fait écho se vivent comme une réalité secondaire avec une toponymie précise, des personnages-avatars connus, replongeant le joueur dans un mythe-monde familier. Pour ce type de fanfics, le contexte et les références de production sont fortement liés au contexte et aux références de réception. Aussi, le récit s'érige-t-il en mémoire collective du jeu, à même de garder la trace du joueur/de son avatar, puisque par ses actions, chaque individu est susceptible de faire évoluer une ou des zones du jeu et de rentrer dans l'histoire (l'Histoire?). 
Le souci de l'historicité n'est pas toujours présent dans la narration ; certaines fanfictions vont valoriser la dimension symbolique plutôt que l'authenticité. Sur les forums, des récits épiques sont bâtis par et pour la guilde ; le vécu y est remanié, rehaussé, tout simplement romancé ${ }^{1}$ pour enorgueillir le(s) héros. Mais, après tout, dans la réalité même, l'Histoire n'a-t-elle pas une part de mythologie? «La culture n'est jamais d'un seul tenant [...] elle concilie des récits qui s'affrontent. [...] mémoire et imagination se mêlent» (Bruner 2002: 111-113). Il y a, à n'en pas douter, une part de "narcissisme » dans la volonté de créer des mythes autour du jeu (Berry et Brougère 2002). Cependant, les récits mythicohistoriques sont avant tout des ressources ethnocentrées; elles s'imposent singulièrement comme supports d'identification, des marqueurs culturels. Les histoires " nous procurent des modèles du monde. [...] partager des histoires, c'est créer une communauté interprétative [...] une communauté culturelle, donner naissance à un ensemble de lois» (Bruner 2002 : 38). En effet, la fanfiction fait loi tout autant qu'elle fait foi (c'est la preuve de l'épreuve commune endurée) ; pour cette raison sans doute est-elle créatrice d'un sentiment de groupe. Le joueur se fabrique un outillage identitaire et une microculture faits d'une communauté, de mythes, de mondes anciens comme autant de strates. Internet est, selon Kaufmann, " un laboratoire de nouveaux modes de définition de soi » (2004: 108) ; présentement, le soi prend la forme d'une évasion dans le simulacre anthropologique, dans une identité anthropoludique.

La fanfiction fait office de mémoire événementielle quand elle relate des (hauts) faits de jeu et, quel que soit par ailleurs son degré d'historicité, elle reste une référence inévitable à l'univers ludique (période, lieux, races...) lui-même inventé : elle est fiction d'une fiction. C'est la raison pour laquelle nous la qualifierons de mythologie mémorielle. Au final, entre l'éphémère présent de l'action et l'éternel présent de l'univers, la narration a valeur de mémoire, une trace du monde et de soi ; elle se démarque encore comme un liant groupal pour une virtualité partagée, comme une fabrique de semblables avec qui fraterniser. En tout état de cause, la fanfiction est, jusqu'ici, dans un rapport de continuation ou de complémentarité avec le jeu. Mais ce n'est pas toujours le cas : le récit peut, dans sa confrontation au jeu, être perçu comme outil de

1. Sur ce terme, qui revient sous la plume des joueurs/écrivains, voir l'analyse dans la seconde partie de l'article. 
résistance, une forme de mise à distance. C'est le propos de la partie suivante.

Selon Roger Caillois (1958: 315), le propre de toute activité ludique est d'être «une création dont le joueur reste maître »; il a notamment «le pouvoir d'interrompre librement l'activité commencée ». La définition ne s'applique guère au jeu multijoueur de type persistant dominé par l'indéterminisme (Natkin 2004) et la multilinéarité. Le MMORPG se déroule en temps réel et pour une durée indéterminée, les objectifs sont indéfinis et a priori incertains quant à leur issue, le jeu est sans fin (proche), sans étapes obligées dans un monde en perpétuelle évolution.

L'activité ludique a pour autres caractéristiques de s'inscrire dans un cadre interactif fortement spatialisé (cartes, niveaux...) et multijoueur. Des milliers de personnes «peuplent» le jeu. Pour accueillir le flot des figurants, les territoires de vie virtuelle sont expansés et ouverts. Chacun des joueurs incarne une destinée parmi une multitude d'autres ; confronté à la contingence, à l'inconstance des guildes, des intentions et des intentionnalités, il trace sa trajectoire d'actions à travers des labyrinthes sans murs. Le jeu est multilinéaire et potentiellement à états infinis; la tension narrative y est incessante parce que l'intrigue ne prend pas fin de façon déterminée. Si l'ouverture des espaces est propice à la libre exploration, les dimensions spatiales donnent un sentiment d'inachevé, sans certitude aucune que tous les lieux du labyrinthe ont été visités. Au-delà de l'intrigue, la tension s'accompagne d'une contrainte de téléprésence forte: on ne peut en effet quitter le jeu librement et trop longtemps sinon au risque de subir les foudres de la guilde ou de perdre de l'expérience par rapport à d'autres.

À tous égards, les MMORPG sont des jeux de la démesure, vertigineux, instables et sur lesquels les joueurs n'ont que peu d'emprise. Les récits des fanfictions symbolisent des espaces de réappropriation, de résistance, qui, en marge du jeu, permettent de reconfigurer et de circonscrire l'espace-temps, de réinventer des mondes, de créer des socialités nouvelles. La temporalité se construit autour de la finitude et de la linéarité du récit; elle rompt avec le caractère persistant et la structure labyrinthique du jeu. A l'échelle de l'histoire, l'espace est d'évidence remanié, borné à un micro-monde, un modèle réduit du monde de référence. Somme toute, la clôture narrative dans sa linéarité, 
sa finitude et sa contigüité exprime un besoin de contrôle sur un jeu à espace et à temps ouverts; il s'agit de retrouver une histoire à chronologie fermée et à locus restreint présente dans les jeux «classiques $»^{2}$. C'est à travers l'inscription narrative que l'espace (indéfini, expansé) devient un ensemble de lieux délimités, précis, parce que le récit donne du relief à l'espace et produit « des géographies d'actions »(Certeau 1980: 171). L'histoire fait la géographie.

Le jeu et le récit s'inscrivent également, nous l'avons vu plus haut, dans une dynamique de socialisation ; ils aident à installer un sentiment communautaire. Les premiers instants de prise en main du jeu donnent déjà lieu à un bricolage identitaire dans le choix de l'avatar et de ses caractéristiques. Le joueur semble s'identifier à son avatar comme à un «second soi » (Turkle 1984), au point de faire sienne une pseudoculture (celle de l'avatar). Le folklore et les mythes créés par le jeu et la fanfic sont des outils d'une presqu'identité raciale (trolls, humains, elfes, gnomes,...) et clanique exprimée dans les guildes. Le schéma d'appréhension du monde, les catégorisations, les enjeux et les protagonistes sont simplifiés. L'altérité est clairement définie (horde contre alliance, bien contre mal, ...). On renoue ici avec la tribalité primitive. Les mythes et les histoires forment à l'échelle de la guilde une micro-culture tribale qui a vocation à expliquer le monde (persistant $\mathrm{du}$ jeu) dans tous ses aspects et tend donc vers la cosmogonie $\mathrm{e}^{3}$ La primitivité se retrouve dans les réalisations picturales (fan art) représentant des créatures humanoïdes marquées de tatouages ou de scarifications. Le fonctionnement même de la guilde renvoie à un modèle social de type tribal de par sa taille et son caractère hiérarchisé.

La pensée magique est une autre manifestation de la « culture sauvage » (Lévi-Strauss 1962) présente dans les MMORPG et leurs fictions : les joueurs peuvent être fascinés par le surnaturel et les âges farouches durant lesquels l'absence de technologie donne tout pouvoir à la magie. Elle témoigne d'un attrait pour un passé de la toute puissance, pour un monde de tous les possibles, à l'image des dessins numérisés «mutants » qui figurent une technologie « animale », des architectures

2. On pense ici aux jeux solo de type FPS ou aux jeux de missions (Counter Strike, Resistance...) aux parcours et passages obligés, aux objectifs déterminés, aux scénarios contraints et immuables.

3. «Au tout début c'était sombre et vide... et puis vint le Gnome ! Depuis la nuit des temps, les Gnomes (...)» extrait de : http://eq2.mondespersistants.com/ ?article $=85$ 
moitié minérales moitié végétales, grotesques, «émotionnelles et douloureuses»(Gerosa 2008). Pour finir, les fanfics évoquent en soi les récits de tradition orale : ceux contés au coin du feu et qui relatent la traque et la capture d'un animal après une chasse glorieuse, un combat mémorable ou quelque excursion en une contrée inconnue.

Qu'ils soient à vocation tribale ou simplement ouverts au toutvenant, les récits faniques se nourrissent immanquablement de la mythologie créée par le jeu, ce qui fait de leurs auteurs des adeptes du «braconnage », liberté que s'accordent les usagers dans les «arts de faire » des pratiques quotidiennes (Certeau 1980) en recourant à la ruse, au détournement et au bricolage. Pour Jenkins, qui qualifie les auteurs de "braconniers textuels ${ }^{4}$ », la réécriture des séries télévisées prend des formes diverses (1992: 162-177) : écriture de scènes inexistantes dans l'œuvre originale, fin différente pour les personnages, centration sur un personnage secondaire de la série, rencontre de deux univers fictionnels (cross-over), scénarios originaux avec des personnes réelles...

Pour ce qui est des jeux persistants, la fanfiction rappelle un assemblage bricolé fait de personnages du jeu, d'univers chaotiques, dystopiques et de compétences sortis des contes fantastiques. Elle est parfois l'occasion de proposer des mythes fondateurs dans un monde en perpétuelle fondation ou des mythes eschatologiques avec des fins choisies. Quoi qu'il en soit, elle est l'œuvre de l'innutrition, entre la copie du plagiaire et la création originale ; son auteur intègre assurément les canons du genre, mais se laisse aller à de l'invention dans le style, les personnages ou les situations : il fait un pot-pourri de morceaux choisis dans des restes de parties, des fragments de mémoire et des surplus d'imagination. Telle est la fanfic, un art «premier» et métisse.

Les concepteurs des MMORPG les plus populaires ${ }^{5}$ (et antérieurement les romans de type médiéval fantastique, en particulier l'œuvre maîtresse de Tolkien) ont trouvé leur inspiration dans une multitude d'histoires moyenâgeuses, de mythes et légendes divers (celtiques ou germaniques par exemple). Il s'ensuit une " composition hétéroclite» (Lévi-Strauss 1962: 26) propre au bricolage, un univers rapiécé. Le monde des MMORPG est donc né d'un syncrétisme forcé,

4 Traduction de « textual poachers ».

5. Dark Age of Camelot, Diablo2, Guild Wars, Ultima, Lineage Online, Everquest ou encore l'incontournable World of Warcraft. 
d'une transculturalité approximative; il continue d'évoluer avec les prolongements bricolés, denses et semble-t-il inépuisables des éditeurs (patchs d'extension, novellisations). De l'avis de Nelson Goodman (cité dans Ong-Van-Cung 2002: 19), «pour construire le monde [...] on démarre toujours avec des mondes déjà à disposition; faire, c'est refaire ». Ce sont précisément ces manières de faire des mondes qu'utilisent les pratiques scripturaires amateurs. Le bricolage ne fait que répondre au bricolage, les éditeurs de jeux ont ouvert la voie à des recréations récréatives, à des jeux d'écriture sans compter.

Nous sommes dans l'ère confuse de l'open source, du piratagepartage, de la culture participative et du Web 2.0 : elle invite au braconnage, au «faire soi-même " (Genvo 2008) et a ouvert la voie à une dictature des anonymes qui prennent la liberté de prendre, de défaire, de recréer. Dans ces conditions, les productions culturelles voient naturellement leurs contenus transfigurés, détournés, réappropriés sur un réseau où s'est installé "un imaginaire commun d'ouverture à l'infini » (Ehrenberg 1995 : 275).

\section{Un paradoxe : une expression libre préprogrammée et récupérée par le jeu}

Comme nous venons de le voir spécifiquement pour les MMORPG et d'une autre manière comme dans l'ensemble des cas des fanfictions, qu'elles s'inspirent de chansons, de mangas, de séries télévisées ou de jeux vidéo, l'écriture personnelle de fictions marque indéniablement une forme de libération des contraintes du jeu tel qu'il est conçu par les développeurs et tel qu'il est joué en société : "Cette section vous est en fait entièrement dédiée. Vous y trouverez des histoires écrites par les joueurs et pour les joueurs. Bien évidemment, vous faites exactement ce que vous voulez. Vous pouvez écrire une histoire totalement imaginaire en plusieurs chapitres (ou un seul si vous le désirez) ou raconter votre soirée d'hier sur Battle.net. L'idéal est évidemment de romancer un peu tout le bazar [nos italiques] ». (http://worldofwarcraft.judgehype.com/ index.php?page=fanfiction). La citation est particulièrement éclairante en ce qu'elle pose comme postulat (et comme évidence) de l'activité d'écriture la liberté du joueur tant sur la matière traitée (imagination ou transcription de parties effectives) que sur la forme ( à propos de la question du nombre de «chapitres », qui rappellent évidemment l'unité de la quête, nous y reviendrons), mais aussi parce qu'elle fixe, tout en 
posant en préalable la liberté, un modèle à atteindre (un «idéal») : « romancer un peu tout le bazar». La métaphore du bazar renvoie clairement au «world» of «warcraft » à la fois comme lieu (ou monde) et comme désordre (en particulier celui du combat et de la guerre); et, se joue, là, comme l'impératif de respecter les qualités propres du jeu (voir la modulation « un peu ») comme espace du conflit (fût-il ludique) ; mais, en même temps, il faut lui donner une forme, celle du roman, c'est-à-dire adopter une double conversion de l'espace (du jeu, de l'arène) en temps (du récit) et du désordre (ou du contingent et du fortuit des combats et du jeu) en nécessité (de l'ordre narratif), que l'on peut qualifier de catharsis au sens propre (Ricœur 1983 : 86-92). Le romancement (http://www.cnrtl.fr/definition/romancer) peut avoir différents sens, qui correspondent à diverses conceptions du roman :

1. soit il renvoie de manière neutre à la forme seule du roman (c'est évidemment un peu vague);

2. soit, plus précisément, il signifie, en référence à un type particulier de romans, "Introduire effectivement dans la réalité [comprendre ici, la réalité de la partie, du jeu] des éléments dignes du roman: l'aventure, l'amour [selon un mode d'écriture très répandu dans les fanfictions!] »; on trouve des typologies sur les sites dédiés à ces activités (par exemple sur http://kirintor-rp.forumsrpg.net/index.htm) qui soulignent ce type d'enrichissement ou d'apport avec la différence entre « aventures et récits» et le «quotidien »;

3. enfin, « romancer ", c'est aussi « Se représenter mentalement telle chose comme plus intéressante, plus belle qu'elle n'est réellement, en estompant certains aspects, en ajoutant des enjolivements "; mais « rendre plus beau ou plus intéressant le jeu » doit se comprendre non seulement comme une revendication de qualités propres pour les fanfictions ou plus généralement pour les fanarts, mais aussi et surtout comme une activité tournée vers l'extérieur de l'écriture, vers l'activité du jeu nourrie, en intérêt et en beauté, par le romancement. En clair, « romancer », ça profite à la fanfic, mais ça profite aussi au jeu.

On voit, donc, dans cette simple citation, se dessiner les contours de notre réflexion sur l'activité d'écriture comme proclamation de liberté, comme affirmation d'une beauté et d'un intérêt en dehors du jeu et paradoxalement comme retour et enrichissement du jeu. Tentons, d'abord de circonscrire la dimension libératrice des activités scripturales.

En écrivant, le joueur prend, donc, de la distance vis-à-vis d'une activité ludique parfois accaparante, développe, par la narration, une 
capacité herméneutique et critique. Certains joueurs se représentent clairement le temps du récit comme une pause dans la continuité du jeu, une délivrance, une évasion : "Pour moi, écrire des fanfictions est vraiment une forme de libération, une évasion temporaire de WOW et le rappel que j'ai d'autres passe-temps et passions que WOW lui-même. C'était surtout le cas quand je gagnais en niveau. Comprenez moi bien, j'aime monter en niveau, mais il y a des moments où je ne suis pas dans le jeu (serveur down, job) ${ }^{6} »$. De ce point de vue, apparaît la différence fondamentale avec les autres types de fanfictions qui viennent, pour leur part, se nicher dans les béances des produits culturels de masse? Dans ces cas-là, l'écriture est interstitielle ; elle vient combler un " trou " dans la représentation initiale (Martin 2007 : 187-188) ; bien sûr, la création autour des MMORPG s'appuie sur une structure de l'action de jeu fondée sur la quête comme unité ou comme fragment constitutif (Walker 2007 : 307-310) : cette totalité narrative, facile à appréhender pour le joueur/rédacteur, semble jouer le même rôle que la chanson ou l'épisode pour d'autres types de productions de fans. Cependant, au contraire des autres fanfictions, pour ce qui concerne les MMORPG, l'écriture constitue un décrochage vis-à-vis de la "persistance " du jeu, une escapade loin des obligations quotidiennes de jouer voire une évasion. Il s'agit, en quelque sorte, à travers elle, sinon de se libérer, du moins de distendre le lien, de rester, certes, lié à l'univers du jeu, mais en n'étant pas dans le jeu: on aura, par exemple, la possibilité de suivre les avancées de sa guilde dans une quête, à travers sa narration en ligne, sans avoir à retourner dans le jeu, si on ne dispose pas du temps nécessaire. Il faut bien considérer que les concepteurs du jeu comblent d'eux-mêmes les éventuelles «discontinuités » du jeu ${ }^{8}$, par exemple

6. «For me, writing fanfiction is really a form of release, a temporary escape from WoW, and a reminder that I do have other hobbies and passions other than WoW itself. This was especially so when I was levelling up. Don't get me wrong, I love levelling up, but there are points where i'm not always in the game (server downtime, my job), and I use those opportunities to enjoy writing fanfiction ». Accessible en ligne : http://frostisthenewblack.blogspot.com/ 2008/07/wow-blogging-and-fanfiction.html

7. L'hétéroclite d'un album de chansons et le délai entre les différents albums, la multiplicité des épisodes d'un «anime » ou des volumes d'un manga, les intersaisons des séries télévisées et la structure interne de chaque épisode forgée par la nécessité des coupures publicitaires.

8. À travers les novellisations autour du jeu, parues en France chez Fleuve noir, par le biais des sites officiels, grâce aux animatiques dans le jeu ou encore par le biais d'une communication personnelle à destination des guildes ou des joueurs. 
lors de lancement d'extensions comme «The Burning Crusade » le 16 janvier 2007 ou «Wrath of the Litch King » le 13 novembre 2008. On perçoit donc combien la fanfic renvoie à des compensations visant à atténuer l'indéterminisme de l'univers ludique ou à prendre ses distances par rapport à celui-ci. L'auteur de récits fait le choix d'actions à l'intérieur d'événements eux-mêmes choisis (l'imprévu est prévu), contrôle tous les personnages, orchestre le jeu d'acteurs, ménage le suspense (sur le mode épisodique par exemple) et a la maîtrise du temps, du rythme, de l'espace. Les déterminismes existentiel (personnages), actanciel (actions) et circonstanciel (espace-temps) sont à la discrétion de l'auteur qui satisfait ainsi son besoin de contrôle. Quant au besoin de distanciation, il transparaît dans la forme du récit : usage d'humour, de second degré ou des commentaires à destination des lecteurs (métalepses) sur le mode des échanges verbaux dans le cadre du jeu.

Ces réflexions laissent à penser que l'appropriation se fait par (ré)interprétations ; les joueurs se sentent parfois prisonniers du monde virtuel et de ses signes, mais ils se veulent libres de leurs significations. Les récits, les poèmes, le fan art, représentent cette part d'interprétation de l'univers. Le fictionnel, pour eux, libère du virtuel.

La prise de distance à travers l'écriture en ligne ne constitue cependant aucunement une rupture franche avec les partenaires de jeu et les concepteurs. Ce qui frappe, s'agissant des produits Blizzard (Diablo, Starcraft ou Warcraft), est l'encadrement par la société de nombreuses pratiques de joueurs en dehors du jeu; le site officiel est pensé dans une lignée web 2.0 (un peu dirigiste) et les développeurs s'expriment souvent (directement ou indirectement) à travers les grands forums « indépendants» (par exemple, knowledgeofwow.com). Au contraire des nombreuses fanfictions portant sur des jeux aussi différents que Sonic, Zelda ou Final Fantasy VIII ${ }^{9}$, les créations liées aux MMORPG et plus singulièrement encore à WOW se trouvent sur les sites officiels ou sur des sites spécialement consacrés à ce jeu et très souvent sur des sites de guildes. C'est que la communauté des joueurs constitue le lieu d'un profond contrôle social, en particulier dans le cadre astreignant des arborescences des sites de guildes (à travers ce qu'elles prévoient d'accueillir ou pas) et dans l'activité de commentaire sur les fanfictions (dans leurs validations, dans l'appel à modifications, dans

9. Les plus grosses entrées sur le site généraliste de fanfictions : http:// www.fanfiction.net/game/. 
l'infléchissement d'une fiction découpée en chapitres...) ; on passe insensiblement de la co-création de contenus inspirés par une passion commune à la censure au nom d'une orthodoxie du jeu. Le rapport au monde « officiel » de Warcraft est le premier critère dans le classement des fanfictions (par exemple sur http://www.wowwiki.com/ WoWWiki:Fan_fiction_guidelines). L'encadrement de l'activité d'écriture dans la guilde ou plus globalement dans la communauté des joueurs maintient une cohérence avec l'univers du jeu et le récit principal tenu par Blizzard dans et hors du jeu; des prescriptions accompagnent, sur les sites, les appels à la création (contrainte); au même titre que les comportements des avatars dans le jeu sont normés ${ }^{10}$, la rédaction de fanfics doit se plier à des règles liées au cadre, à l'histoire générale, aux personnages, à leurs caractéristiques propres et à leurs rapports, etc. Plus inattendu, peut-être, le rappel fréquent des grands principes narratifs (par exemple sur http://forums.wow-europe.com/ thread.html?topicId=99890149\&sid=2) autour du schéma actanciel, de la structure du récit, de la focalisation fait apparaître clairement un impératif : il faut se nourrir des "formes classiques de la narration » (Salmon 2008) ; au contraire de "la disparition des histoires" qu'annonce Christian Salmon, selon lequel « la capacité d'Hollywood à raconter une histoire serait progressivement grignotée par l'expansion des messages et des microrécits dans la médiasphère. Le récit traditionnel avec suspense, conflit et résolution serait en passe d'être noyé dans le bruit universel et le désordre visuel », nous assistons à un appel au renforcement des structures traditionnelles de récit, comme s'il s'agissait d'un besoin essentiel pour les joueurs. C'est ce qui doit nous intéresser maintenant.

Ce sont, à notre sens, les qualités narratives respectives des fanfictions et des MMORPG qui expliquent l'injonction à respecter des règles strictes dans le cadre des activités d'écriture satellites de l'occupation ludique. Si l'on veut bien se référer au concept bakhtinien de chronotope, très efficace dans l'appréhension des types de récit (Bakhtine 1975), apparaissent très clairement deux complexes spatiaux, temporels et émotifs (384), l'un nouveau qui est celui du monde de "world of warcraft », l'autre traditionnel, lié en particulier aux romans de chevalerie, la route (385-387). Or, l'espace en expansion que

10. Il existe une véritable éthique commune en particulier vis-à-vis des «newbies » et de véritables codes de conduite dans les guildes, d'autant plus nécessaires qu'ils sont la clé de la réussite dans les quêtes. 
constitue WOW nous paraît difficile à appréhender non seulement en tant que récit, mais encore en tant que jeu tant qu'il n'a pas été exploré par des itinéraires, tant qu'il n'a pas été transformé en lieu anthropologique (Augé 1992: 149), c'est-à-dire en un espace où s'inscrivent l'identité, les relations et l'histoire de ceux qui l'habitent. On le conçoit aisément pour certains types de joueurs comme les «socializers» et les « explorers» (Bartle 1996); mais cela est tout aussi vrai pour les «killers» et les « achievers », qui seront limités dans leurs objectifs propres s'ils ne parviennent pas à développer des stratégies fondées sur un savoir encyclopédique du jeu, sur les caractéristiques des avatars, sur les modes de fonctionnement des guildes, sur les phases d'expansion du jeu. Or, la quête identitaire, la demande relationnelle et l'historisation de soi et des autres passent, dans WOW, par la mise en récit (sur des modes différents allant du simple dialogue vocal dans le jeu jusqu'aux plus complexes fanfictions) ; dans cet effort commun pour faire tenir ce monde ou pour rendre cet espace praticable par des usages sociaux, pour y fonder le lieu d'un jeu social, la tradition livresque de l'heroïc fantasy, les novellisations centrées sur World of Warcraft et les fanfictions jouent un rôle essentiel, en ce qu'elles préfigurent et reconfigurent les actions de jeu, au même titre que les récits, de manière générale, structurent nos actions "IRL ", dans le monde réel (sic) : « le texte est un ensemble d'instructions que le lecteur individuel ou le public exécutent de façon passive ou créatrice » (Ricœur 1983 : 146). La spirale de la triple mimèsis décrite par Ricœur (1. précompréhension du monde de l'action, de sa sémantique, de sa symbolique et de sa temporalité ; 2 . mise en intrigue, configuration ; 3. restitution au temps de l'agir) nous permet de comprendre comment une culture narrative partagée prépare l'activité ludique. Un contreexemple peut nous éclairer sur ce point : dans son récit de l'expérience de «Mankind », Patrick Schmoll souligne combien, au lancement du jeu, « les joueurs sont [...] arrivés dans un univers qui était d'une certaine manière vide d'enjeu, qui n'était qu'un décor, en contradiction avec les traditions et les règles du jeu vidéo (un jeu, une histoire précise, un chemin à parcourir impérativement, des niveaux à passer)» (2008: 72). Force est de constater le succès limité d'un MMOG sans véritable assise narrative, à rapprocher, sur un mode mineur, de l'échec du « deuxième monde » de Canal + ou du feu de paille "2nd life », tous deux sans référence à un espace imaginaire potentiellement narratif, au contraire de l'univers de science-fiction de «Mankind» clairement lié à un certain nombre d'intrigues bien intégrées par le public et à ce titre 
générateur narratif et ludique (voir http://www.mk-histoires.ovh.org/). Puisque le but des concepteurs de WOW est de rendre le jeu le plus praticable et le plus pratiqué possible (c'est-à-dire clairement de multiplier les abonnements, source de profit), il est impératif pour eux d'inciter à la narration et d'organiser ces activités dans le cadre de l'expansion du jeu et de son univers. Le «monde » de Warcraft doit se nourrir de cette matière (au sens aussi où l'on parle de matière de Bretagne pour désigner le cycle arthurien), doit phagocyter tout développement narratif sur la base culturelle du jeu, doit faire ventre de sa propre progéniture : il a besoin de l'activité fabulatrice de ses usagers pour faire croître sa présence sur le réseau ; d'un certain point de vue, nous sommes proches, là, d'une forme de marketing viral tel qu'il se développe aux confins du jeu, du récit et de l'énigme dans les «Alternate Reality Games» (Martin 2008). Il est significatif de voir que ce qui peut servir le jeu est favorisé, alors que ce qui n'est pas directement assimilable est poursuivi au nom de la propriété intellectuelle ${ }^{11}$.

\section{Conclusion}

Dans le cadre des MMORPG, la fanfiction est, d'abord, apparue comme un lieu d'imprégnation socioculturelle, un hommage du joueur à l'univers ludique. A première vue, fortement couplée au jeu, elle constitue, avec lui, une forme d'évasion d'une réalité sociale empreinte d'incertitude. Cependant, la fanfic peut aussi s'opposer au jeu dans la distance qu'elle prend vis-à-vis de ce dernier : elle rompt avec la continuité de l'univers et recrée une nécessaire contiguïté. Entre résilience à la réalité et résistance au jeu, le récit fanique se particularise manifestement en tant que pratique de l'entre-deux, possiblement même porteuse de paradoxes ou de contradictions. Dans le jeu et le récit, l'entre-deux transparaît à différents niveaux d'analyse.

Dans le rapport réalité-fiction : entre un schéma virtuel dystopique (monde chaotique, désordres architectural, biologique, social) et une réalité sans utopie, incertaine ou encore entre monde mythicofictionnel et historicité des actions de jeu relatées.

Dans les alternatives antinomiques qu'offrent les jeux eux-mêmes : entre bien et mal, humanité et animalité, Alliance et Horde ...

11. On pense aux machinimas, les courts-métrages utilisant les moteurs graphiques des jeux. 
Dans la navigation entre modernité du dispositif technique du joueur, symbole de la rationalité, et âges pré-technologiques auxquels il est fait référence dans les jeux. La barbarie et les puissances occultes figurent quant à elles la pensée magique, les régressions ancestrale et enfantine.

Dans le bricolage qui est à l'œuvre dans le récit : entre libre expression (invention individuelle) et obligation de canonicité étendue. Outre les règles du récit, la fanfiction doit respecter celles de l'univers du jeu (atmosphères, personnages, ...) notamment sous l'influence du public lecteur qui exerce un contrôle de type panoptique.

En ce qui concerne ce dernier point, l'œuvre individuelle, orientée par le collectif, l'est également par les ressources narratives à l'origine des jeux. Les éditeurs tolèrent le braconnage narratif et la diversité des « trajectoires d'usage » (Proulx 2002) parce qu'ils ne remettent pas en cause l'unité du jeu. À l'inverse, les fanfictions profiteraient même au jeu qui, inexorablement, fait ventre de tout en utilisant les récits comme des extensions officieuses propices à son expansion.

Plongé dans le magma multimédia et le brouhaha virtuel, l'auteur de récits doit s'intégrer sans disparaître et s'affirmer sans s'exclure ; il est encore une fois dans un entre-deux. 


\section{Références}

Augé, Marc, 1992, Non-lieux. Introduction à une anthropologie de la surmodernité. Paris, Seuil.

Bakhtine, Mikhail, 1975, Esthétique du roman. Paris. Gallimard.

Bartle, Richard A.,1996, Hearts, Clubs, Diamonds, Spades : Players Who suit Muds. Accessible en ligne : http://www.mud.co.uk/richard/ hods.htm

Berry, Vincent et Gilles Brougère, 2002, Play and Virtual Communities on Internet. Accessible en ligne : http://www.get-telecom.fr/archive/ 77/ActesBerry.pdf

Caillois, Roger, 1958, Les Jeux et les hommes. Le Masque et le vertige. Paris, Gallimard.

Certeau, Michel de, 1980, Arts de faire. Paris, Gallimard.

Chibout, Karim et Martial Martin, 2009, à paraître). "Militaires en mobilité : web 2.0 et construction de l'identité ». In Fred Dervin et Yasmine Abbas (dir.), Nouvelles technologies du soi, mobilités et (co) constructions identitaires. Berne, Peter Lang Publishing Group.

Ehrenberg, Alain, 1995, L'Individu incertain. Paris : Hachette Littératures : 1999.

Genvo, Sébastien, 2008, «Comprendre les différentes formes de "faire soi-même" dans les jeux vidéo ». Accessible en ligne : http:// www.ludologique.com/publis/articles_en_ligne.html

Jenkins, H. (1992). Textual Poachers. New York/London : Routledge. Kaufmann, Jean-Claude, 2004, L'Invention de soi. Une théorie de l'identité. Paris, Hachette.

Lévi-Strauss, Claude, 1962, La Pensée sauvage. Paris, Plon.

Maffesoli, Michel, 1988, Le Temps des tribus. Le Déclin de l'individualisme dans les sociétés de masse. Paris, Méridiens-Klincksieck.

Martin, Martial, 2007, "Les séries télévisées sur Internet. Le cas des "fanfictions" ", Médiamorphoses hors Série $3:$ 186-189.

Martin, Martial, 2008, "L'Irruption d'une nouvelle forme narrative : les "alternate reality games" ». In Alexandra Saemmer et Monique Maza (dir.), e-formes : 45-58. Saint-Etienne, Presses Universitaires de Saint-Etienne.

Natkin, Stéphane, 2004, Jeux vidéo et médias du XXIe siècle. Paris, Vuibert. Ong-Van-Cung, Kim Sang, 2002, Descartes et l'ambivalence de la création. Paris, Vrin.

Proulx, Serge, 2002, "Trajectoires d'usages des technologies de communication. Les Formes d'appropriation d'une culture 
numérique comme enjeu d'une société du savoir », Annales des télécommunications 57 (3-4): 180-189.

Ricœur, Paul, 1983, Temps et récit I, Paris, Seuil.

Schmoll, Patrick, 2008, "Communautés de joueurs et "mondes persistants" », Médiamorphoses 22 : 68-75.

Turkle, Sherry, 1984, The Second Self. New York: Simon and Shuster. Walker, Jill, 2007, "A Network of Quests in World of Warcraft ». In Pat Harrigan et Noah Wardrip-Fruin (dir.), Second Person: Role Playing and Story in Games and Playable Media: 307-310. Cambridge: MIT Press. 